\title{
Mesenchymal stem cells: a new strategy for immunosuppression and tissue repair
}

\author{
Yufang Shi ${ }^{1}$, Gangzheng $\mathrm{Hu}^{1}$, Juanjuan $\mathrm{Su}^{1}$, Wenzhao $\mathrm{Li}^{1}$, Qing Chen ${ }^{1}$, Peishun Shou ${ }^{1}$, Chunliang Xu${ }^{1}$,
} Xiaodong Chen ${ }^{1}$, Yin Huang ${ }^{1}$, Zhexin Zhu ${ }^{1}$, Xin Huang ${ }^{1}$, Xiaoyan Han ${ }^{1}$, Ningxia Xie ${ }^{1}$, Guangwen Ren ${ }^{2}$

${ }^{I}$ Key Laboratory of Stem Cell Biology, Institute of Health Sciences, Shanghai Institutes for Biological Sciences, Chinese Academy of Sciences/Shanghai Jiao Tong University School of Medicine, Shanghai 200025, China; ${ }^{2}$ Department of Molecular Genetics, Microbiology and Immunology, Robert Wood Johnson Medical School-University of Medicine and Dentistry of New Jersey, Piscataway, NJ 08854, USA

Mesenchymal stem cells (MSCs) have great potential for treating various diseases, especially those related to tissue damage involving immune reactions. Various studies have demonstrated that MSCs are strongly immunosuppressive in vitro and in vivo. Our recent studies have shown that un-stimulated MSCs are indeed incapable of immunosuppression; they become potently immunosuppressive upon stimulation with the supernatant of activated lymphocytes, or with combinations of IFN- $\gamma$ with TNF- $\alpha$, IL-1 $\alpha$ or IL-1 $\beta$. This observation revealed that under certain circumstances, inflammatory cytokines can actually become immunosuppressive. We showed that there is a species variation in the mechanisms of MSC-mediated immunosuppression: immunosuppression by cytokine-primed mouse MSCs is mediated by nitric oxide (NO), whereas immunosuppression by cytokine-primed human MSCs is executed through indoleamine 2, 3-dioxygenase (IDO). Additionally, upon stimulation with the inflammatory cytokines, both mouse and human MSCs secrete several leukocyte chemokines that apparently serve to attract immune cells into the proximity with MSCs, where NO or IDO is predicted to be most active. Therefore, immunosuppression by inflammatory cytokine-stimulated MSCs occurs via the concerted action of chemokines and immune-inhibitory NO or IDO produced by MSCs. Thus, our results provide novel information about the mechanisms of MSC-mediated immunosuppression and for better application of MSCs in treating tissue injuries induced by immune responses.

Keywords: MSCs, immunosuppression, tissue repair, immune diseases

Cell Research (2010) 20:510-518. doi:10.1038/cr.2010.44; published online 6 April 2010

\section{Mesenchymal stem cells}

Tissue mesenchymal stem cells (MSCs) or MSC-like cells exist in almost all tissues, such as bone marrow, muscle, fat, hair follicles, tooth root, placenta, brain periosteum, dermis, perichondrium, umbilical cord, Wharton's jelly, lung, liver and spleen [1]. They were initially identified by their potential of induced differentiation into several mesenchymal lineages: bone, cartilage, adipose tissue, muscle and tendon [2]. However, recently it is shown that some types of MSC-like cells isolated from both human and mouse can be induced into cells of

Correspondence: Yufang Shi

Tel: +86-21-63848329; Fax: +86-21-63852655

E-mail: shiyufang2@gmail.com endodermal and neuroectodermal lineages, including endothelium, neurons and hepatocytes [3-7]. Intravenously infused MSCs immigrate to a variety of organs, with the priority to injured or inflammatory tissues $[8,9]$.

Bone marrow stem cells can be classified as either $\mathrm{CD}^{+} 4^{+}$or $\mathrm{CD} 34^{-}$; $\mathrm{CD} 34^{+}$cells give rise to blood cells while $\mathrm{CD} 34^{-}$cells (and even $\mathrm{CD} 34^{+}$cells) are believed to be more primitive and can differentiate into many cell types. The most intensely studied MSCs are those derived from the adult bone marrow [10]. MSCs are low in frequency in the bone marrow; plating studies indicated that MSCs represented perhaps $0.001-0.01 \%$ of the nucleated cells, which is approximately 10 -fold less abundant than hematopoietic stem cells (HSCs). Human bone marrow-derived MSCs can be cloned and expanded in vitro more than a million-fold and still retain the ability to differentiate into several cell lineages. Thus, it is 
possible to produce sufficiently large numbers of MSCs for cell therapy from a single modest bone marrow aspirate. The most important properties of MSCs are their readiness to grow in culture and their ability to suppress the immune system. In fact, cultured MSCs have already been infused into human subjects for safety studies and for early clinical testing to support bone marrow transplantation, and for the treatment of diseases like graftversus-host diseases (GvHD), osteogenesis imperfecta, and glycogen storage disease, demonstrating great promise clinically. In a recent study in monkeys, MSCs expanded ex vivo were found to redistribute into many different tissues following transfusion and were identifiable after 9-21 months following transfusion. MSCs settled mostly in tissues with a high turnover rate, such as gastrointestinal tissues, kidney, lung, liver, thymus and skin [11]. Both autologous and allogeneic MSCs became similarly distributed. It has also been reported that MSCs preferentially localize to the wounded or damaged tissue sites.

\section{Mesenchymal stem cells in wound repair}

The wide tissue distribution and multipotent differentiation of MSCs, together with the observed reparative effects of infused MSCs in many clinical and preclinical models [10-25], strongly suggest a critical role of MSCs in injury healing. Though there has been no direct evidence of migration path of MSCs to the wound site, due to the lack of a reliable marker for MSCs, it is reasonable to assume that severe tissue damage may mobilize and recruit remote MSCs to the injured sites [26]. These recruited and resident MSCs regulate the repair process by differentiation into several kinds of stromal and/or damaged cell types, as well as by providing a microenvironment through the interaction with many types of tissue and immune cells, such as fibroblasts, endothelial and epithelial cells, macrophages, neutrophils and lymphocytes (Figure 1). This interaction is believed to be critical in providing a microenvironment for tissue regeneration and wound repair. This critical research area is expected to attract great deal of attention of biologists in the near future.

Cell replacement and interaction with fibroblasts, endothelial and epithelial cells

Based on the anatomic sites and etiology, tissue injury can be roughly classified into the following types: epithelial-lined organs and non-epithelial-lined organs, immunity-mediated and non-immunity-mediated. Epi-

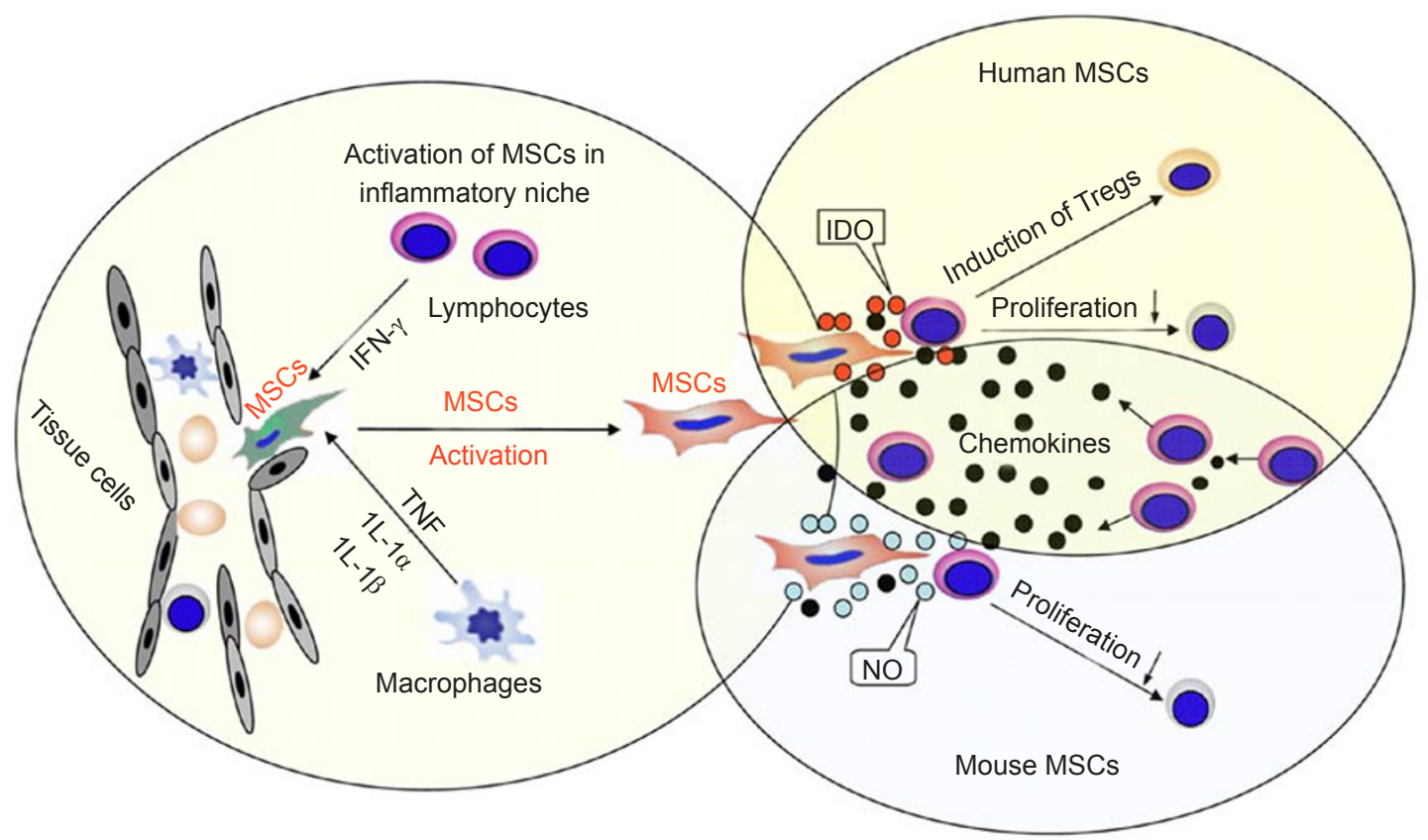

Figure 1 Activation of MSCs in inflammatory niche and immunosuppression on lymphocytes. The recruited MSCs are readily activated by inflammatory cytokines produced by activated lymphocytes and monocytes, and release a lot of chemokines, and NO or IDO. Lymphocytes are attracted in close proximity to MSCs by chemokines, where mouse MSCs inhibit activation/ proliferation of lymphocytes by releasing NO, but human MSCs inhibit proliferation of lymphocytes and/or induce T regulatory cells (Tregs) by producing IDO. 
thelial-lined organs mainly include skin, gut, lung and cornea, being characterized by direct contact with the external environment and a myriad of microbes. Immunitymediated injury may result from autoimmune diseases or infection of pathogens or allogeneic transplantation. Physical and chemical factors and ischemia are the common cause of non-immunity-mediated tissue injury. The mechanism underlying the effect of MSCs on injury repair may differ for different injury types. As for the injury of bone, cartilage, muscle and tendon caused by mechanical force and osteogenesis imperfecta, differentiation and replacement of the damaged cells may be the main mechanisms.

Fibroblasts and endothelial cells may be the most commonly involved cell types during the process of injury repair. Fibroblasts are the main source of extracellular matrix, and contribute to the remodeling of damaged tissues. Expansion of endothelial cells controls the speed of angiogenesis. MSCs from close vicinity or bone marrow are believed to migrate into the damaged tissue sites and release an array of growth factors, such as epidermal growth factor (EGF), fibroblast growth factor (FGF), platelet-derived growth factor (PDGF), transforming growth factor- $\beta$ (TGF- $\beta$ ), vascular endothelial growth factor (VEGF), insulin-like growth factor 1 (IGF-1), angiopoietin-1 and stromal cell-derived factor-1 (SDF-1), all of which may influence the development of fibroblasts and endothelial cells [27]. This may be the fundamental mechanisms generally used by the MSCs for enhancing repair of all kinds of injury. Indeed, the MSCs disappeared shortly after infusion in the injured tissues, and the differentiation into damaged cell types is a rare event in injuries, like myocardial infarction $[28,29]$ and acute renal failure [15]. In fact, supernatant from MSCs was effective in treating skin-excision wound $[18,30]$.

\section{Inflammatory niche and interaction with immune cells}

In a healthy body, pathogenic tissue injury, to a great extent, involves activation of certain immune cells. Apoptotic cells are silently cleared by the resident macrophages without causing inflammation. However, acute tissue damage, even when resulted from non-immune or non-infectious factors, is usually followed by inflammation, because certain cell components released from the necrotic cells and microvasculature damage always lead to enhanced vasopermeability and the infiltration of macrophages and neutrophils; phagocytosis of necrotic cells by the resident and infiltrated macrophages and neutrophils always results in the release of inflammatory factors, such as TNF- $\alpha$, IL-1, chemokines, leukotrienes and free radicals. The immunity (or infection)-mediated tissue injury may be more complex and involves many kinds of immune cells, such as B cells, $\mathrm{CD} 4^{+}$and $\mathrm{CD} 8^{+}$ $\mathrm{T}$ cells and natural killer cells (NK cells). Epithelial damage in epithelial-lined organs makes the tissue exposed to a myriad of microbes, and the role of immune cells in the repair process is essential and regulated more delicately.

Clearance of damaged tissue and invaded pathogens make immune cells a non-redundant participator in injury healing, nevertheless, excessive activation of immune cells inevitably interferes with repair, or even aggravates injury. The poor immunogenicity and active immunosuppressive capacity of MSCs have been well documented. Therefore, the reparative function of MSCs observed in so many injury models may be, at least partly, attributed to the immune inhibitory function. Besides the suppression on lymphocytes, dendritic cells (DCs) and NK cells in immunity-mediated injury, the interaction of MSCs with macrophages and neutrophils may be especially important in certain types of injury [31]. Macrophages normally reside in almost all tissues, being ready for clearing damaged tissue cells or invaded pathogens. Macrophages have different activation pathways and functional statuses. For example, macrophages classically activated by IFN- $\gamma$ plus LPS are featured by respiratory burst, secretion of many pro-inflammatory cytokines and production of nitric oxide (NO), thus promoting cellular immunity to intracellular pathogens and causing tissue damage. On the contrary, alternative activation stimulated by IL-4 or IL-13 promotes humoral immunity to parasites and tissue repair [32]. MSCs were observed to promote immigration of macrophages into skin-wound bed [18]. MSCs activated by LPS or TNF- $\alpha$ reprogrammed macrophages by releasing prostaglandin E-2 (PGE-2), and attenuated sepsis [33]. Inhibition of respiratory burst of neutrophils may be partially responsible for the beneficial effects of MSCs in the model of myocardial infarction, as the accumulated neutrophils might release reactive oxygen species and proteolytic enzymes, propagating injury to surrounding myocytes $[34,35]$.

The inflammatory cells and factors are commonly present in injured sites, and the inflammatory niche may be an important prerequisite for MSCs to fulfil the reparative function in most cases. This proposal is based on two observations: (1) infused MSCs preferentially immigrated into inflammatory sites, probably due to the inflammatory cell-produced chemokines and inflammatory factor-induced upregulation of chemokine receptors on MSCs [36]; (2) the immunosuppression potential of MSCs is not natural, and requires the induction by inflammatory factors (IFN- $\gamma /$ TNF- $\alpha$ ) [37] (Figure 1). Therefore, the fine kinetics of the interactions between MSCs and inflammatory and immune factors is critical for the outcome of wound healing. 
The microenvironment of MSC-mediated immunosuppression

The immunosuppression property of MSCs has attracted much attention of immunologists, stem cell biologists and clinicians. However, detailed investigations of how the microenvironment affects immunosuppression of MSCs are still lacking. Microenvironment should be carefully considered when using MSCs in the development of novel therapeutic strategies for critical diseases such as suppression of GvHD in patients post bone marrow transplantation. Cell-to-cell contact and soluble factors are thought to be key aspects of MSCs-mediated immunosuppression.

\section{Cell-cell interaction}

MSCs and T cells The PD-1 pathway was shown to be involved in MSCs-mediated immunomodulation by cellto-cell contact [38], while TGF- $\alpha 1$, hepatocyte growth factor (HGF), PGE-2, soluble HLA-G5, heme oxygenase (HO)-1, indoleamine 2,3-dioxygenase (IDO) and inducible nitric-oxide synthase (iNOS) [39-42], are molecules responsible for the immunomodulation through humoral factors. The soluble factors are produced by MSCs constitutively or released after MSCs' cross-talk with target cells. It is important to point out that the factors responsible for suppressing $T$ cells vary depending on the experimental system and in vivo situations.

Recently, we found that the immunosuppressive function of MSCs was elicited by IFN- $\gamma$ and the concomitant presence of any of the three other proinflammatory cytokines, TNF- $\alpha$, IL- $1 \alpha$ or IL- $1 \beta$ in mice [37]. These cytokine combinations provoke the expression of high levels of several chemokines and iNOS by MSCs (Figure 1). Chemokines drive $T$ cell migration into the proximity with MSCs, where T cell response was controlled by NO (Figure 1). MSCs have already been shown to produce several chemokines [43], which may serve to recruit lymphocytes. In our experiment, MSCs expressed very high levels of several leukocyte chemokines, most notably CXCL9, CXCL10 and CXCL11, which were ligands for the T cell-specific chemokine receptor, CXCR3. Cytokine-activated MSCs also attracted bone marrowderived DCs, macrophages, as well as B cells [37].

MSCs and B cells MSCs also inhibit B lymphocyte proliferation, which is partially due to the physical contact between MSCs and B cells, partially mediated by PD-1/ PD-L1, -L2, and partially due to soluble factors released by MSCs in culture supernatants. However, culture conditions could drastically change the immunomodulatory effects of MSCs on activation of B lymphocytes [44].
MSCs and NK cells Similar to what was observed in T cells, soluble factors such as TGF- $\beta 1$ and PGE-2 played an important role in the MSC-mediated suppression of NK cell proliferation [45]. Several studies showed that IL-2-activated NK cells (but not freshly isolated NK cells) efficiently lysed autologous and allogeneic MSCs. The activating NK cell receptors, NKp30, NKG2D and DNAM-1 were the major receptors responsible for the NK cell-mediated cytotoxicity against MSCs. Meanwhile, MSCs expressed ligands for these activating NK receptors: ULBPs, PVR and Nectin-2 [46]. It has been demonstrated that human MSCs inhibited IL-2- or IL-15driven NK cell proliferation [46].

MSCs and APCs MSCs inhibit the maturation of monocyte-derived myeloid DCs by down-regulation of the expression of MHC and costimulatory molecules, such as CD40, CD86, and CD83 [47] and the production of IL-12 upon Toll-like receptor (TLR)-mediated DC activation [48]. After interaction with MSCs, myeloid originated DCs produce a decreased amount of TNF- $\alpha$, while plasmacytoid DCs produce an increased amount of IL-10 and TNF- $\alpha$ which play important roles in DC maturation, migration and presentation of antigen. The mechanism of MSCs-induced inhibition of DC maturation, differentiation and function was demonstrated to be mediated by soluble factors, such as PGE-2, released upon cell-to-cell contact [49].

While human MSCs could promote the superantigeninduced activation of purified T cells, addition of antigenpresenting cells (APCs, either monocytes or DCs) to the cultures inhibited the T cell responses. This contact- and dose-dependent inhibition is accompanied by secretion of large quantities of IL-10 and aberrant APC maturation [50].

MSCs and soluble factors Recently, soluble factors such as chemotactic factors, TNF- $\alpha$, IL- $1 \beta$ and TGF- $\alpha$ have been actively examined for their effects on MSCs.

The migration of MSCs to the sites of injury or inflammation is mediated by chemotactic factors produced by immune cells. It has been demonstrated that human MSCs showed significant chemotaxis responses to several factors, including PDGF, VEGF, IGF-1, IL-8, bone morphogenetic protein (BMP)-4 and BMP-7 [51].

One of the major cytokines released at sites of injury is TNF- $\alpha$, which is a key regulator of the NF- $\kappa B$ pathway. NF- $\kappa \mathrm{B}$ pathway plays an important role in regulating genes that influence cell migration, proliferation, differentiation and inflammation. Bocker et al. showed that TNF- $\alpha$ strongly increased the migratory ability of human MSCs through regulating the extracellular ma- 
trix. NF- $\kappa \mathrm{B}$ target genes which influence migration, such as vascular cell adhesion molecule-1, CD44 and matrix metalloproteinase 9 were upregulated by TNF- $\alpha$. Meanwhile, TNF- $\alpha$ also stimulated the proliferation of human MSCs by upregulating expression of the proliferationassociated gene, cyclin D1 [52].

Osteogenic differentiation from the MSCs was suppressed by IL- $1 \beta$ and TNF- $\alpha$. Both cytokines inhibited the differentiation-associated increase in alkaline phosphatase (ALP) activity and the upregulation of gene expression of ALP, procollagen, runt-related transcription factor 2 (Runx2) and osterix. Thus, the potential involvement of inflammatory mediators need to be taken into consideration in achieving optimal repair of bone and other tissues with MSCs [53].

TGF- $\alpha$ is an important mediator of wound healing and the injury response. Human MSCs release VEGF as a beneficial paracrine response. It has been demonstrated that low-dose TGF- $\alpha(500 \mathrm{pg} / \mathrm{ml})$ suppressed the production of VEGF by MSCs, while high-dose TGF- $\alpha$ (250 ng/ $\mathrm{ml})$ significantly upregulated the production of VEGF. High-dose TGF- $\alpha$ also increased TNF- $\alpha$-stimulated VEGF production by MSCs [54]. Therefore, the concerted actions of various cytokines in the microenvironment play critical roles in modulating the physiological properties of MSCs.

\section{The application of MSCs in treating immune disor- ders}

Preclinical and clinical models of diseases for MSC applications

Since Bartholomew and co-worker in 2002, first used a baboon model to reveal that injection of allogeneic MSCs prolonged skin-graft survival in primates [55] (although the immunosuppressive effect on allogeneic skin graft was only brief), more than 12 preclinical animal models have been developed to demonstrate the capabilities of MSCs, especially in modulating immune responses during the pathological processes of diseases [56]. Recent advancement has focused on the crucial roles of MSCs in regulating tolerance against allograft rejection and GvHD [57], due to its profound immunomodulatory effects such as the inhibition of donor $\mathrm{T}$ cell reactivity to histocomapatiblity antigens of the normal tissues of recipient. Our published data demostrated that IFN and NO production were essential for MSC-mediated immunosuppression in GvHD model [37], but not TGF- $\beta$ [58], and PGE-2 [49]. MSCs were also shown to have a potential therapeutic effect against infiltration of $\mathrm{T}$ cells in central nervous system (CNS) in experimental autoimmune encephalomyelitis (EAE), a model of multiple scle- rosis (MS) induced by myelin oligodendrocyte glycoprotein (MOG) [20]. Furthermore, systemic administration of MSCs was found to decrease the in vivo production of proteolipid protein (PLP) peptide-specific antibodies in the EAE model [59]. In mouse rheumatoid arthritis (RA) model, systematically injected-MSCs inhibited specific T cell response and also decreased the production of proinflammatory cytokines such as IFN, meanwhile regulatory T cells were induced by MSCs to suppress the inflammation [60]. Aside from the immunoregulation of MSCs, other important therapeutic effects of MSCs shown in some models such as myocardial infarction, diabetes and acute renal failure, were mainly to support the regeneration of, or differentiation into specific cells of the injured tissues [56, 61].

Medically, MSCs have been used clinically for a decade, mainly in the field of treating life-threatening conditions such as severe, acute GvHD, especially after HSC transplantation [57]. Researches into the role that MSCs play in clinical applications have focused on their characteristics. Besides the ability to differentiate into special tissues of their origins, MSCs can also provide support for the growth and differentiation of haemopoietic progenitor cells in the bone marrow microenvironment and promote engraftment of haemopoietic cells. Furthermore, MSCs suppress proliferation of activated lymphocytes in a non-human leukocyte antigen (HLA)-restricted manner [62]. Since 2008, a phase II clinical trial using cotransplantation of HLA-identical culture-expanded sibling MSCs with HLA-identical sibling HSCs in patients with hematologic malignancy have been in process. No patients had adverse effects during or immediately after infusions of MSCs, and nine patients showed improvements, indicating that this therapeutic strategy could be promising clinically [63]. It was exciting that in the phase I clinical studies in 2007, partial liver cirrhosis patients infused with MSCs from peripheral vein showed improvement, and in 2008, USA Food and Drugs Administration (FDA) gave green light on clinical trials for application of MSCs in MS and articular cartilage repair, which could become potential clinical strategies in the future $[64,65]$.

\section{Engraftment of MSCs}

It is of note that in all of these preclinical and clinical studies, the engraftment of MSCs into damaged tissues via migration to enhance tissue repair/regeneration is a crucial process for clinical efficacy, regardless of the types of organs with specific diseases. Earlier, in vivo studies with MSCs focused mainly on their ability to facilitate the engraftment of transplanted HSCs [66]. As more and more clinical studies are performed, 
the engraftment properties of MSCs were gradually evaluated in many models and clinical trials. In 2000, a study using human MSCs transplanted in utero in sheep demonstrated that there was long-term engraftment as long as 13 months after transplantation even when cells were transplanted after the expected development of immunocompetence, and transplanted human MSCs could undergo site-specific differentiation into chondrocytes, adipocytes, myocytes and cardiomyocytes, bone marrow stromal cells and thymic stroma [67]. To date, in preclinical models, wound sites and proinflammatory environments were found capable of enhancing the engraftment of MSCs such as in lung fibrosis in mice induced by bleomycin [68]. However, the overwhelming majority of MSCs were found in lung after systemic administration in normal recipients, and after a while, these MSCs disappeared gradually [69]. The mechanisms of these phenomena are still unclear. Recent studies suggested that the allogenicity of MSCs had no significant effect on the engraftment of MSCs in wound healing [30], which could probably solve the problem of MSC sources in clinical applications.

The site of delivery probably affects the trafficking of MSCs to target organs. Generally, two approaches of systemic administration have been used for MSC applications. One is intravenous (IV) injection such as injection from peripheral vein (tail vein in mice), utilizing the capabilities of MSCs to migrate to specific inflammatory tissues in vivo including cartilage, liver, lung and so on $[70,71]$. The engraftment was demonstrated in animal models and capable of persisting as long as 13 months after transplantation [67]. The other is local intraarterial (IA) injection which can enhance the accumulation and increase the dose of MSCs in injured tissues. In a 2007 phase I study of application of MSCs for treatment of liver cirrhosis, patients were injected with human MSCs from liver arteries [65]. Besides the different methods of administration, the timing of delivery and number of cells delivered are also very important. Both higher numbers of MSCs and early phase delivery have been found to improve engraftment rates after the presentation of ischemia [72], and the protocol had been optimized [73].

Factors and molecules which play very important roles in MSCs engraftment in vivo have been discovered. For instance, integrin- $\beta 1$ was shown to be necessary for MSC myocardial migration and engraftment [74]. Furthermore, the Wnt modulator Sfrp2 could also enhance MSC engraftment [75]. It is well known that clinical use requires the delivery of adequate numbers of MSCs to specific sites for a therapeutic effect, so further studies are necessary for enhancing the engraftment of MSCs in specific diseases. For example, due to the low expression of CXCR4 on MSCs, CXCR4 transduction with retrovirus has been used to enhance the engraftment of MSCs via increasing cell invasion in response to SDF-1, which is typically present in inflammatory environments as the ligand for CXCR4 [76]. Another approach involved the conjugation of antibodies to the cell surface through protein $\mathrm{G}$ or protein $\mathrm{A}$, which increased the rates of migration via antibodies bearing an accessible Fc region [77]. How to increase the efficiency of engraftment is the key for clinical applications of MSCs. Through engineered approaches, it is possible that better therapeutic effects could be achieved.

\section{Time of MSC administration}

Muriel Sudres et al. found in their study that MSCs failed to prevent GvHD in mouse, and the failure was not due to MSC rejection [78]. However, we have found that MSCs can prolong mice survival in GvHD mouse model [37]. One difference between these two studies was the infusion time of MSCs. Muriel Sudres et al. injected MSCs 10-15 min before GvHD induction, whereas we injected MSCs 3 days and 7 days after bone marrow transplantation. It is possible that the time of MSC administration is important for the therapeutic effect. Based on the above discussion that the immunosuppression ability of MSCs must be induced by inflammatory cytokines, it is conceivable that administration of MSCs at the peak of inflammation may improve the treatment effect. However, this hypothesis needs to be further tested.

Enhancing the immunosuppression and engraftment of MSCs by pretreatment with inflammatory cytokines

Previous studies have suggested that pretreatment of MSCs with inflammatory cytokines may enhance their immunosuppressive ability as well as engraftment in vivo. As mentioned above, MSC-mediated immunosuppression is not innate, but rather it is induced by inflammatory cytokines, such as IFN- $\gamma$, TNF- $\alpha$ and IL$1 \beta$. When treated with inflammatory cytokines, it was confirmed that, both in vitro and in vivo, mouse MSCs expressed high levels of iNOS and chemokines, which were essential for the immunosuppression effect [37]. NO production by treated MSCs lasted for several days after treatment withdrawal (unpublished data), which implied that treated MSCs could elicit the immunosuppression without the requirement for continuous presence of inflammatory cytokines. We recently found that MSCs, when treated with inflammatory cytokines, could up-regulate ICAM-1 and VCAM-1, which were important for T cell adhesion. Antibodies against ICAM-1 and VCAM-1 reduced T cell accumulation around MSCs and significantly reversed suppression of proliferation and 
cytokine production by activated T cells (unpublished data). Therefore, their "education" of MSCs with inflammatory cytokines before infusion may improve its immunosuppressive function in vivo.

Previous studies have implied that pretreatment of MSCs with inflammatory cytokines may enhance MSC homing and engraftment, thus improving therapeutic effects. Upon treatment with inflammatory cytokines, like TNF- $\alpha$, TGF- $\beta 1$ and IL- $1 \beta$, MSCs up-regulate the expression of MMP2, MT1-MMP and/or MMP9, which promote their directed migration across reconstituted human basal membrane in vitro [79]. Another group led by Matthias Schieker found that TNF- $\alpha$ could strongly augment human MSC migration through extracellular matrix and this effect was regulated, at least in part, by the NF- $\mathrm{kB}$ pathway [52]. CXCR4 was suggested to play an important role in the engraftment of MSCs into the bone marrow [80], whereas MSCs used integrin- $\beta 1$ for myocardial migration and engraftment [74]. MSCs can up-regulate both intracellular and membrane expression of CXCR4 after a short pretreatment with cytokines, gaining enhanced engraftment ability to the bone marrow of irradiated NOD/SCID mice, and transplantation of cytokines-treated MSCs results in faster hematological recovery [74]. Another recent study showed that TNF- $\alpha$ pretreatment before transplantation could increase MSC engraftment efficiency and improve recovery of cardiac function after cardiac infarction [81].

Thus, cytokine-pretreatment can enhance the immunosuppressive effect as well as MSC engraftment in some settings. We hypothesize that pretreatment by inflammatory cytokines should be a better strategy for future application of MSCs in clinic.

\section{Acknowledgments}

The research in the authors' laboratory is supported by grants from the Ministry of Science and Technology of China (2010CB945600), Knowledge Innovation Project of the Chinese Academy of Science (KSCX1-YW-22) and the National Science and Technology Project of China (2009ZX09503-024).

\section{References}

1 Bianco P, Robey PG, Simmons PJ. Mesenchymal stem cells: revisiting history, concepts, and assays. Cell Stem Cell 2008; 2:313-319.

2 Pittenger MF, Mackay AM, Beck SC, et al. Multilineage potential of adult human mesenchymal stem cells. Science 1999; 284:143-147.

3 Jiang Y, Jahagirdar BN, Reinhardt RL, et al. Pluripotency of mesenchymal stem cells derived from adult marrow. Nature 2002; 418:41-49.

4 Sasaki M, Abe R, Fujita Y, et al. Mesenchymal stem cells are recruited into wounded skin and contribute to wound repair by transdifferentiation into multiple skin cell type. J Immunol 2008; 180:2581-2587.

5 Fernandes KJ, McKenzie IA, Mill P, et al. A dermal niche for multipotent adult skin-derived precursor cells. Nat Cell Biol 2004; 6:1082-1093.

6 Sanchez-Ramos J, Song S, Cardozo-Pelaez F, et al. Adult bone marrow stromal cells differentiate into neural cells in vitro. Exp Neurol 2000; 164:247-256.

7 Schwartz RE, Reyes M, Koodie L, et al. Multipotent adult progenitor cells from bone marrow differentiate into functional hepatocyte-like cells. J Clin Invest 2002; 109:1291-1302.

8 Devine SM, Cobbs C, Jennings M, et al. Mesenchymal stem cells distribute to a wide range of tissues following systemic infusion into nonhuman primates. Blood 2003; 101:2999-3001.

9 Kawada H, Fujita J, Kinjo K, et al. Nonhematopoietic mesenchymal stem cells can be mobilized and differentiate into cardiomyocytes after myocardial infarction. Blood 2004; 104:3581-3587.

10 Tzaribachev N, Vaegler M, Schaefer J, et al. Mesenchymal stromal cells: a novel treatment option for steroid-induced avascular osteonecrosis. Isr Med Assoc J 2008; 10:232-234.

11 Richardson SM, Hoyland JA. Stem cell regeneration of degenerated intervertebral discs: current status. Curr Pain Headache Rep 2008; 12:83-88.

12 Amado LC, Saliaris AP, Schuleri KH, et al. Cardiac repair with intramyocardial injection of allogeneic mesenchymal stem cells after myocardial infarction. Proc Natl Acad Sci USA 2005; 102:11474-11479.

13 Lee JW, Fang X, Gupta N, et al. Allogeneic human mesenchymal stem cells for treatment of E. coli endotoxin-induced acute lung injury in the ex vivo perfused human lung. Proc Natl Acad Sci USA 2009; 106:16357-16362.

14 Parekkadan B, van Poll D, Suganuma K, et al. Mesenchymal stem cell-derived molecules reverse fulminant hepatic failure. PLoS One 2007; 2:e941.

15 Togel F, Hu Z, Weiss K, et al. Administered mesenchymal stem cells protect against ischemic acute renal failure through differentiation-independent mechanisms. Am J Physiol Renal Physiol 2005; 289:F31-F42.

16 Kim JM, Lee ST, Chu K, et al. Systemic transplantation of human adipose stem cells attenuated cerebral inflammation and degeneration in a hemorrhagic stroke model. Brain Res 2007; 1183:43-50.

17 Inoue Y, Iriyama A, Ueno S, et al. Subretinal transplantation of bone marrow mesenchymal stem cells delays retinal degeneration in the RCS rat model of retinal degeneration. Exp Eye Res 2007; 85:234-241.

18 Chen L, Tredget EE, Wu PY, et al. Paracrine factors of mesenchymal stem cells recruit macrophages and endothelial lineage cells and enhance wound healing. PLoS One 2008; 3:e1886.

19 Hayashi Y, Tsuji S, Tsujii M, et al. Topical implantation of mesenchymal stem cells has beneficial effects on healing of experimental colitis in rats. J Pharmacol Exp Ther 2008; 326:523-531.

20 Zappia E, Casazza S, Pedemonte E, et al. Mesenchymal stem cells ameliorate experimental autoimmune encephalomyelitis inducing T-cell anergy. Blood 2005; 106:1755-1761.

21 Horwitz EM, Prockop DJ, Fitzpatrick LA, et al. Transplant- 
ability and therapeutic effects of bone marrow-derived mesenchymal cells in children with osteogenesis imperfecta. Nat Med 1999; 5:309-313.

22 Garcia-Olmo D, Garcia-Arranz M, Herreros D, et al. A phase I clinical trial of the treatment of Crohn's fistula by adipose mesenchymal stem cell transplantation. Dis Colon Rectum 2005; 48:1416-1423.

23 Le Blanc K, Rasmusson I, Sundberg B, et al. Treatment of severe acute graft-versus-host disease with third party haploidentical mesenchymal stem cells. Lancet 2004; 363:1439-1441.

24 Noth U, Steinert AF, Tuan RS. Technology insight: adult mesenchymal stem cells for osteoarthritis therapy. Nat Clin Pract Rheumatol 2008; 4:371-380.

25 Erokhin VV, Vasil'eva IA, Konopliannikov AG, et al. Systemic transplantation of autologous mesenchymal stem cells of the bone marrow in the treatment of patients with multidrugresistant pulmonary tuberculosis. Probl Tuberk Bolezn Legk 2008; 3-6.

26 Karp JM, Leng Teo GS. Mesenchymal stem cell homing: the devil is in the details. Cell Stem Cell 2009; 4:206-216.

27 da Silva Meirelles L, Fontes AM, Covas DT, et al. Mechanisms involved in the therapeutic properties of mesenchymal stem cells. Cytokine Growth Factor Rev 2009; 20:419-427.

28 Kinnaird T, Stabile E, Burnett MS, et al. Local delivery of marrow-derived stromal cells augments collateral perfusion through paracrine mechanisms. Circulation 2004; 109:15431549 .

29 Gnecchi M, He H, Liang OD, et al. Paracrine action accounts for marked protection of ischemic heart by Akt-modified mesenchymal stem cells. Nat Med 2005; 11:367-368.

30 Chen L, Tredget EE, Liu C, et al. Analysis of allogenicity of mesenchymal stem cells in engraftment and wound healing in mice. PLoS One 2009; 4:e7119.

31 Seno H, Miyoshi H, Brown SL, et al. Efficient colonic mucosal wound repair requires Trem2 signaling. Proc Natl Acad Sci USA 2009; 106:256-261.

32 Gordon S. Alternative activation of macrophages. Nat Rev Immunol 2003; 3:23-35.

33 Nemeth K, Leelahavanichkul A, Yuen PS, et al. Bone marrow stromal cells attenuate sepsis via prostaglandin E(2)-dependent reprogramming of host macrophages to increase their interleukin-10 production. Nat Med 2009; 15:42-49.

34 Raffaghello L, Bianchi G, Bertolotto M, et al. Human mesenchymal stem cells inhibit neutrophil apoptosis: a model for neutrophil preservation in the bone marrow niche. Stem Cells 2008; 26:151-162.

35 Frangogiannis N. The immune system and cardiac repair. Pharmacol Res 2008; 58:88-111.

36 Ponte AL, Marais E, Gallay N, et al. The in vitro migration capacity of human bone marrow mesenchymal stem cells: comparison of chemokine and growth factor chemotactic activities. Stem Cells 2007; 25:1737-1745.

37 Ren GW, Zhang LY, Zhao X, et al. Mesenchymal stem cellmediated immunosuppression occurs via concerted action of chemokines and nitric oxide. Cell Stem Cell 2008; 2:141-150.

38 Augello A, Tasso R, Negrini SM, et al. Bone marrow mesenchymal progenitor cells inhibit lymphocyte proliferation by activation of the programmed death 1 pathway. Eur J Immunol 2005; 35:1482-1490.
39 Chabannes D, Hill M, Merieau E, et al. A role for heme oxygenase-1 in the immunosuppressive effect of adult rat and human mesenchymal stem cells. Blood 2007; 110:3691-3694.

40 Jones B J, Brooke G, Atkinson K, et al. Immunosuppression by placental indoleamine 2,3-dioxygenase: a role for mesenchymal stem cells. Placenta 2007; 28:1174-1181.

41 Sato K, Ozaki K, Oh I, et al. Nitric oxide plays a critical role in suppression of T-cell proliferation by mesenchymal stem cells. Blood 2007; 109:228-234.

42 Selmani Z, Naji A, Zidi I, et al. Human leukocyte antigen G5 secretion by human mesenchymal stem cells is required to suppess T lymphocyte and natural killer function and to induce CD4+CD25 high FOXP3+ regulatory T cells. Stem Cells 2008; 26:212-222.

43 Chamberlain G, Fox J, Ashton B, et al. Concise review: Mesenchymal stem cells: their phenotype, differentiation capacity, immunological features, and potential for homing. Stem Cells 2007; 25:2739-2749.

44 Corcione A, Benvenuto F, Ferretti E, et al. Human mesenchymal stem cells modulate B-cell functions. Blood 2006; 107:367-372.

45 Sotiropoulou PA, Perez SA, Gritzapis AD, et al. Interactions between human mesenchymal stem cells and natural killer cells. Stem Cells 2006; 24:74-85.

46 Spaggiari GM, Capobianco A, Becchetti S, Mingari MC, Moretta L. Mesenchymal stem cell-natural killer cell interactions: evidence that activate NK cells are capable of killing MSCs, whereas MSCs can inhibit IL-2-induced NK-cell proliferation. Blood 2006; 2107:1484-1489.

47 Zhang W, Ge W, Li C, et al. Effects of mesenchymal stem cells on differentiation, maturation, and function of human monocyte-derived dendritic cells. Stem Cells Dev 2004; 13:263-271.

48 Jiang XX, Zhang Y, Liu B, et al. Human mesenchymal stem cells inhibit differentiation and function of monocyte-derived dendritic cells. Blood 2005; 105:4120-4126.

49 Aggarwal S, Pittenger MF. Human mesenchymal stem cells modulate allogeneic immune cell responses. Blood 2005; 105:1815-1822.

50 Beyth S, Borovsky Z, Mevorach D, et al. Human mesenchymal stem cells alter antigen-presenting cell maturation and induce T-cell unresponsiveness. Blood 2005; 105:2214-2219.

51 Mishima Y, Lotz M. Chemotaxis of human articular chondrocytes and mesenchymal stem cells. J Orthop Res 2008; 26:1407-1412.

52 Bocker W, Docheva D, Prall WC, et al. IKK-2 is required for NF-alpha-induced invasion and proliferation of human mesenchymal stem cells. J Mol Med 2008; 86:1183-1192.

53 Lacey D, Park D, Short B, et al. TNF and IL-1 inhibits osteogenic differentiation of mouse mesenchymal stem cells in vitro: implications for bone repair during chronic inflammation. Inflamm Res 2007; 2007:S392-S393.

54 Wang Y, Crisostomo PR, Wang M, Markel TA, Novotny NM, Meldrum DR. TGF-alpha increases human mesenchymal stem cell-secreted VEGF by MEK- and PI3-K- but not JNKor ERK-dependent mechanisms. Am J Physiol Regul Integr Comp Physiol 2008; 95:R1115-R1123.

55 Bartholomew A, Sturgeon C, Siatskas M, et al. Mesenchymal stem cells suppress lymphocyte proliferation in vitro and prolong skin graft survival in vivo. Exp Hematol 2002; 30:42-48. 
56 Uccelli A, Moretta L, Pistoia V. Mesenchymal stem cells in health and disease. Nat Rev Immunol 2008; 8:726-736.

57 Le Blanc K, Ringden O. Mesenchymal stem cells: properties and role in clinical bone marrow transplantation. Curr Opin Immunol 2006; 18:586-591.

58 Groh ME, Maitra B, Szekely E, et al. Human mesenchymal stem cells require monocyte-mediated activation to suppress alloreactive T cells. Exp Hematol 2005; 33:928-934.

59 Gerdoni E, Gallo B, Casazza S, et al. Mesenchymal stem cells effectively modulate pathogenic immune response in experimental autoimmune encephalomyelitis. Ann Neurol 2007; 61:219-227.

60 Chen FH, Tuan RS. Mesenchymal stem cells in arthritic diseases. Arthritis Res Ther 2008; 10:223.

61 Tang JM, Xie QY, Pan GD, et al. Mesenchymal stem cells participate in angiogenesis and improve heart function in rat model of myocardial ischemia with reperfusion. Eur J Cardiothorac Surg 2006; 30:353-361.

62 Ning H, Yang F, Jiang M, et al. The correlation between cotransplantation of mesenchymal stem cells and higher recurrence rate in hematologic malignancy patients: outcome of a pilot clinical study. Leukemia 2008; 22:593-599.

63 Le Blanc K, Frassoni F, Ball L, et al. Mesenchymal stem cells for treatment of steroid-resistant, severe, acute graft-versushost disease: a phase II study. Lancet 2008; 371:1579-1586.

64 Mohamadnejad M, Alimoghaddam K, Mohyeddin-Bonab M, et al. Phase 1 trial of autologous bone marrow mesenchymal stem cell transplantation in patients with decompensated liver cirrhosis. Arch Iran Med 2007; 10:459-466.

65 Mohamadnejad M, Namiri M, Bagheri M, et al. Phase 1 human trial of autologous bone marrow-hematopoietic stem cell transplantation in patients with decompensated cirrhosis. World J Gastroenterol 2007; 13:3359-3363.

66 Noort WA, Kruisselbrink AB, in’t Anker PS, et al. Mesenchymal stem cells promote engraftment of human umbilical cord blood-derived CD34(+) cells in NOD/SCID mice. Exp Hematol 2002; 30:870-878.

67 Liechty KW, MacKenzie TC, Shaaban AF, et al. Human mesenchymal stem cells engraft and demonstrate sites-specific differentiation after in utero transplantation in sheep. Nat Med 2000; 6:1282-1286.

68 Luis A, Ortiz FG, Christine M, et al. Mesenchymal stem cell engraftment in lung is enhanced in response to bleomycin exposure and ameliorates its fibrotic effects. Proc Natl Acad Sci USA 2003; 100:8407-8411.

69 Lama VN, Smith L, Badri L, et al. Evidence for tissue-resident mesenchymal stem cells in human adult lung from studies of transplanted allografts. J Clin Invest 2007; 117:989-996.

70 Sakaida I, Terai S, Yamamoto N, et al. Transplantation of bone marrow cells reduces CCl4-induced liver fibrosis in mice. $\mathrm{He}$ patology 2004; 40:1304-1311.

71 Chen JL, Li Y, Wang L, et al. Therapeutic benefit of intravenous administration of bone marrow stromal cells after cerebral ischemia in rats. Stroke 2001; 32:1005-1011.

72 Omori Y, Honmou O, Harada K, et al. Optimization of a therapeutic protocol for intravenous injection of human mesenchymal stem cells after cerebral ischemia in adult rats. Brain Res 2008; 1236:30-38.

73 Chen CP, Liu SH, Huang JP, et al. Engraftment potential of human placenta-derived mesenchymal stem cells after in utero transplantation in rats. Hum Reprod 2009; 24:154-165.

74 Ip JE, Wu Y, Huang J, Zhang L, Pratt RE, Dzau VJ. Mesenchymal stem cells use integrin betal not CXC chemokine receptor 4 for myocardial migration and engraftment. Mol Biol Cell 2007; 18:2873-2882.

75 Alfaro MP, Pagni M, Vincent A, et al. The Wnt modulator sFRP2 enhances mesenchymal stem cell engraftment, granulation tissue formation and myocardial repair. Proc Natl Acad Sci USA 2008; 105:18366-18371.

76 Cheng Z, Ou L, Zhou X, et al. Targeted migration of mesenchymal stem cells modified with CXCR4 gene to infarcted myocardium improves cardiac performance. Mol Ther 2008; 16:571-579.

77 Dennis JE, Cohen N, Goldberg VM, Caplan AI. Targeted delivery of progenitor cells for cartilage repair. J Orthop Res 2004; 22:735-741.

78 Sudres M, Norol F, Trenado A, et al. Bone marrow mesenchymal stem cells suppress lymphocyte proliferation in vitro but fail to prevent graft-versus-host disease in mice. J Immunol 2006; 176:7761-7767.

79 Ries C, Egea V, Karow M, et al. MMP-2, MT1-MMP, and TIMP-2 are essential for the invasive capacity of human mesenchymal stem cells: differential regulation by inflammatory cytokines. Blood 2007; 109:4055-4063.

80 Shi M, Li J, Liao L, et al. Regulation of CXCR4 expression in human mesenchymal stem cells by cytokine treatment: role in homing efficiency in NOD/SCID mice. Haematologica 2007; 92:897-904.

81 Kim YS, Park HJ, Hong MH, et al. TNF-alpha enhances engraftment of mesenchymal stem cells into infarcted myocardium. Front Biosci 2009; 14:2845-2856. 